\title{
SPACECRAFT DESCENT AND TRANSLATION IN THE SMALL-BODY FIXED FRAME
}

\author{
Stephen B. Broschart*and Daniel J. Scheeres ${ }^{\dagger}$ \\ University of Michigan, Ann Arbor, MI, USA
}

\begin{abstract}
The dynamics of spacecraft descent and translation near small, irregularly-shaped bodies, such as comets and asteroids, is investigated. Through linearization, we derive a constant thrust solution for general translation in the body-fixed frame. We improve this nominal result through error estimation and numerically show the resulting control to be very accurate despite the non-linear gravitational potential. We also present the notion of a free-drop manifold from which a spacecraft will arrive at a given target without thrust. Sensitivity of our controller to parameter uncertainty is presented. Numerical covariance results are given for descents to three realistic asteroid shapes.
\end{abstract}

\section{Motivation}

Recently, the world's space agencies have been planning and executing missions to small celestial bodies, such as asteroids and comets. It is thought that the pristine composition of these bodies could lend insight into how our solar system was formed. However, missions to these irregularly shaped bodies are challenging due to their remoteness and complex gravitational fields.

In this paper, we consider the problem of spacecraft translation in an arbitrary gravitational field such as that near a small-body. Descent is a subset of translation such that the destination lies on the surface of the body. To date, the only successful descent to an asteroid surface was done by NASA's Near Earth Asteroid Rendezvous (NEAR) mission team using a series of open-loop thrusts to de-orbit the spacecraft. ${ }^{1}$ Theoretical work on descent to small bodies has taken a number of approaches. Cui and Cui ${ }^{2}$ developed a method for descent using feedback control on altitude and thrust modulation. Guelman and Harel ${ }^{3}$ gave an optimal, electric propulsion descent solution from orbit for the spherical body case. The Japan Aerospace Exploration Agency's Hayabusa sample return mission, currently en route to asteroid Itokawa, plans on using optical navigation feedback, an altimeter, and a target marker to descend to the surface. ${ }^{4}$ Here, we will develop an open-loop, general translation solution that uses constant thrust to move the spacecraft in the body-fixed frame.

We will first formulate the problem and derive a general linearized dynamics solution. This general solution is used to find the particular solutions for free-drop and constant thrust dynamics. We then estimate the error associated with the linearization and use it to improve controller performance. Examples of translation performance above asteroids Itokawa and Eros are given. We proceed to look at the sensitivity of controller performance to uncertainty in the system parameters and give covariance results for descent maneuvers above models of the asteroids Itokawa, Eros, and Vesta. Finally, a descent scenario to asteroid Eros is described in detail.

\section{Problem Formulation}

In this work, we will assume that the small-body has uniform density and rotates uniformly about the principal axis corresponding to its maximum moment of inertia $(z)$. We will further assume that the

\footnotetext{
${ }^{*}$ Ph.D. Candidate, Department of Aerospace Engineering, 1320 Beal Ave., Ann Arbor, MI 48109, USA, AIAA Student Member, sbroscha@engin.umich.edu

$\dagger$ Associate Professor, Department of Aerospace Engineering, Associate Fellow of the AIAA

Copyright (C) 2004 by Stephen B. Broschart and Daniel J. Scheeres
} 
spacecraft has an internal model of the asteroid shape and estimates of rotation state and density. The equations of motion in the body-fixed, rotating, Cartesian frame with origin at the center of mass are:

$$
\dot{\mathbf{X}}=A \mathbf{X}+\left[\begin{array}{c}
0 \\
0 \\
0 \\
\frac{\partial V}{\partial x}+T_{x} \\
\frac{\partial V}{\partial y}+T_{y} \\
\frac{\partial V}{\partial z}+T_{z}
\end{array}\right]
$$

where

$$
A=\left[\begin{array}{cccccc}
0 & 0 & 0 & 1 & 0 & 0 \\
0 & 0 & 0 & 0 & 1 & 0 \\
0 & 0 & 0 & 0 & 0 & 1 \\
\omega^{2} & 0 & 0 & 0 & 2 \omega & 0 \\
0 & \omega^{2} & 0 & -2 \omega & 0 & 0 \\
0 & 0 & 0 & 0 & 0 & 0
\end{array}\right]
$$

and

$$
\mathbf{X}=\left[\mathbf{r}^{T}, \dot{\mathbf{r}}^{T}\right]^{T}=\left[\begin{array}{llllll}
x, & y, & z, & \dot{x}, & \dot{y}, & \dot{z}
\end{array}\right]^{T}
$$

Here, $\omega$ is the body's rotation rate, $\mathbf{T}$ is the spacecraft thrust vector (with components in each Cartesian direction noted by subscript), and $V$ is the gravitational potential. The problem of translation is addressed here as an interception problem; given an initial state and transfer time, determine the necessary maneuvers to arrive at a specified position regardless of final velocity.

\section{General Linearized Result}

The equations of motion (Eqn. 1) are linear except for the attraction terms derived from the potential. We linearize the attraction vector about the nominal initial position to obtain the following linear equations of motion:

$$
\dot{\mathbf{X}}=(A+\delta) \mathbf{X}+\mathbf{u}
$$

where

$$
\delta=\left[\begin{array}{cc}
0_{3 x 3}, & 0_{3 x 3} \\
\left.\frac{\partial^{2} V}{\partial \mathbf{r}^{2}}\right|_{0}, & 0_{3 x 3}
\end{array}\right]
$$

and

$$
\mathbf{u}=\left[0,0,0,\left(\mathbf{T}+\left.\frac{\partial V}{\partial \mathbf{r}}\right|_{0}-\left.\frac{\partial^{2} V}{\partial \mathbf{r}^{2}}\right|_{0} \mathbf{r}_{0}\right)^{T}\right]^{T},
$$

The general solution for this system of equations is:

$$
\mathbf{X}(t)=e^{(A+\delta) t} \mathbf{X}(0)+\int_{0}^{t} e^{(A+\delta)(t-\tau)} \mathbf{u} d \tau
$$

If the spacecraft thrust, $\mathbf{T}$, is constant (i.e., $\mathbf{u}$ is constant) and the matrix $\left(\left.\frac{\partial^{2} V}{\partial \mathbf{r}^{2}}\right|_{0}+\operatorname{diag}\left(\omega^{2}, \omega^{2}, 0\right)\right)$ is invertible, the following identity holds:

$$
\int_{0}^{t} e^{(A+\delta)(t-\tau)} \mathbf{u} d \tau=(A+\delta)^{-1}\left[e^{(A+\delta) t}-I_{6 x 6}\right] \mathbf{u}
$$


which gives us the following closed form solution for the state at time $t_{f}$ :

$$
\mathbf{X}\left(t_{f}\right)=e^{(A+\delta) t_{f}} \mathbf{X}(0)+(A+\delta)^{-1}\left[e^{(A+\delta) t_{f}}-I_{6 x 6}\right] \mathbf{u}
$$

Here, we have six linear equations with sixteen potential variables (initial state, final state, time of transfer $\left(t_{f}\right)$, and thrust). Given a set of known variables, this formulation can be cast as a solution for a number of different situations. We are presenting solutions to the free-drop and constant thrust translation problems.

\section{Free-drop Solution}

For a mission studying small-body composition, it is desirable to cut off spacecraft thrusters before reaching the surface to avoid contamination. Because of the small gravitational accelerations induced by these bodies, unpowered 'free-drop' descents are possible without excessive impact velocity. By rearranging the general linearized result (Eqn. 5), we can define the locus of initial states that result in impact at a given point without thrust.

$$
\mathbf{X}_{\mathbf{0}}=e^{-(A+\delta) t_{f}}\left(\mathbf{X}_{\mathbf{f}}-(A+\delta)^{-1}\left(e^{(A+\delta) t_{f}}-I_{6 x 6}\right)\left[0_{1 x 3},\left.\frac{\partial V}{\partial \mathbf{r}}\right|_{\mathbf{r}_{\mathbf{f}}} ^{T}-\left.\mathbf{r}_{\mathbf{f}}^{T} \frac{\partial^{2} V}{\partial \mathbf{r}^{2}}\right|_{\mathbf{r}_{\mathbf{f}}} ^{T}\right]^{T}\right)
$$

In this problem, we specify the target position, thrust (zero), and time of transfer. If we neglect touchdown velocity, we have three equations and six unknowns. This defines a three dimensional linear space of initial states in six dimensional phase space that result in touchdown at the target in the given time. If we specify an initial velocity (zero for instance), we can define a line of qualifying initial positions parameterized by $t_{f}$. The manifold of initial states defined by the free-drop solution is useful as a target space for the preceeding transfers.

\section{Constant Thrust Solution}

The general interception problem can be solved by applying a constant thrust in our general linearized result. In this formulation, we are given an initial state, a final position, and a transfer time and asked for the appropriate thrust to complete the transfer. By rearranging Eqn. 5, we find the necessary thrust (Eqn. 7) and linear estimate of the resulting final velocity (Eqn. 8).

$$
\begin{gathered}
\mathbf{T}=G(4: 6,:)\left(\mathbf{X}_{\mathbf{f}}-e^{(A+\delta) t_{f}} \mathbf{X}_{\mathbf{0}}\right)-\left.\frac{\partial V}{\partial \mathbf{r}}\right|_{0}+\left.\frac{\partial^{2} V}{\partial \mathbf{r}^{2}}\right|_{0} \mathbf{r}_{\mathbf{0}} \\
\dot{\mathbf{r}}\left(t_{f}\right)=G(1: 3,4: 6)^{-1}\left(G(1: 3,:) e^{(A+\delta) t_{f}} \mathbf{X}_{\mathbf{0}}-G(1: 3,1: 3) \mathbf{r}\left(t_{f}\right)\right)
\end{gathered}
$$

where

$$
G=\left[e^{(A+\delta) t_{f}}-I_{6 x 6}\right]^{-1}(A+\delta)
$$

The notation $G(a: b, c: d)$ represents the $a$-th through $b$-th rows and $c$-th through $d$-th columns of matrix $G$ (similar to that used in Matlab). A colon alone indicates all rows or columns are included. This thrust solution completes the transfer exactly in the linearized system. Errors arise in the true system due to the nonlinearity of the potential field.

\section{A. Estimate of Linear Error}

We can calculate the error induced by nonlinearity of the potential in the constant thrust solution by comparing the linearized dynamics with the true dynamics. The solution to the non-linear equations of motion (Eqn. 1) is:

$$
\mathbf{X}^{*}(t)=\left[\mathbf{r}^{* T}(t), \dot{\mathbf{r}}^{*^{T}}(t)\right]^{T}=e^{A t} \mathbf{X}(0)+\int_{0}^{t} e^{A(t-\tau)}\left[0_{1 x 3},\left.\quad \frac{\partial V}{\partial \mathbf{r}}\right|_{\mathbf{r}^{*}(t)} ^{T}+\mathbf{T}^{T}\right]^{T} d \tau
$$


Error is determined by subtracting the general linearized solution (Eqn. 5).

$$
\begin{aligned}
\mathbf{E}(t)= & \mathbf{X}^{*}(t)-\mathbf{X}(t) \\
= & e^{A t}\left(I_{6 x 6}-e^{\delta t}\right) \mathbf{X}_{\mathbf{0}}+e^{A t} \int_{0}^{t} e^{-A \tau}\left[\begin{array}{c}
0_{3 x 1} \\
\left.\frac{\partial V}{\partial \mathbf{r}}\right|_{\mathbf{r}^{*}(t)}+\mathbf{T}
\end{array}\right] d \tau \\
& -(A+\delta)^{-1}\left(e^{(A+\delta) t}-I_{6 x 6}\right)\left[\begin{array}{c}
0_{3 x 1} \\
\left.\frac{\partial V}{\partial \mathbf{r}}\right|_{0}+\mathbf{T}-\left.\frac{\partial^{2} V}{\partial \mathbf{r}^{2}}\right|_{0} \mathbf{r}_{\mathbf{0}}
\end{array}\right]
\end{aligned}
$$

Because of the integral involving the true path of the spacecraft, we can not evaluate the error without explicitly integrating the non-linear equations of motion. However, if we assume that the nominal constant thrust solution derived from the linear dynamics is 'close' to the actual path, we can evaluate the integral along the linearized path to obtain a suitable estimate.

If we further assume that all paths near the true path have similar $\frac{\partial V}{\partial \mathbf{r}}$ profiles over time, then we can assume the error vector is nearly the same since the integral is the only path dependent part of the error. If the magnitude of our error is sufficiently small, we can choose a phantom target, defined in Eqn. 11, such that if we use Eqn. 7 to calculate thrust to arrive at the phantom target, we invoke the same error, and therefore, arrive at the desired target.

$$
\mathbf{X}_{\text {phantom }}=\mathbf{X}_{\text {target }}-\mathbf{E}\left(t_{f}\right)
$$

This method also yields an improved estimate of the final velocity by adding the velocity error in Eqn. 10 with the predicted velocity in Eqn. 8.

Using this error estimate approach, we have made our original controller more accurate without numerically integrating the equations of motion. Further, we maintain a closed form solution for the necessary transfer thrust and a well-defined nominal trajectory.

\section{B. Accuracy of Translation Controller}

Using an appropriate phantom target, our constant thrust controller is shown to be very accurate for translations inside a fairly large domain around the initial position. An idea of the limits of the accurate domain can be obtained by considering the case of translations in a point-mass potential field. Our erroneous assumption is that the attraction vector changes in a linear manner as we deviate from the initial position. In actuality, for a change of position in the radial direction, percentage error in this approximation grows as a cubic of the ratio of initial radius to final radius, $\gamma$. If we travel a line of constant radius along either a constant latitude or longitude line, we find that the attraction vector changes as the cosine function. This suggests our linearity assumption is only valid for translations that involve small changes in latitude or longitude. Generally, translations across the same distance are more accurate further from the body since both the angular deviation and $\gamma$ change more slowly. This type of analysis closely predicts the error in the original controller formulation (Eqn. 7) before the error estimate is included. However, the qualitative properties of the error remain the same for the phantom target formulation; the domain of accurate translation is bounded by a maximum allowable angular deviation and a maximum radial deviation.

Contour plots in figures 1 and 2 show numerical results for the nominal miss distances (difference between the target and numerically integrated final position) associated with a class of translations above asteroids Itokawa and Eros, respectively. Figure 1 shows the nominal miss distance, in centimeters, for translations from a stationary initial position $(300,0,-150) m$ (denoted by ' + ') to targets in Itokawa's XZ plane with a transfer time of 1200 seconds. We assume a rotation period of 12.132 hours and density of $2.5 \mathrm{~g} / \mathrm{cm}^{3}$. Our controller misses the desired target by less than 8 centimeters for the translations on the order of a few hundred meters shown here. Figure 2 shows translations to points in the plane $z=5 \mathrm{~km}$ from the initial position $(16,0,5) \mathrm{km}$ above asteroid Eros with rotation period of 5.27 hours and density $3.0 \mathrm{~g} / \mathrm{cm}^{3} .7$ Errors here are on the order of a few centimeters for translation distances on the order of a kilometer. These examples are typical of the miss distances and domain of accuracy seen for this controller in general.

It should be noted that the time of transfer must be kept adequately small to ensure accuracy of the control. If the time of transfer becomes too large, the actual path will deviate from the predicted linearized path and our error estimate will be inaccurate. 




Figure 1. Miss distance $(\mathrm{cm})$ for translation in the $\mathrm{XZ}$ plane near asteroid Itokawa

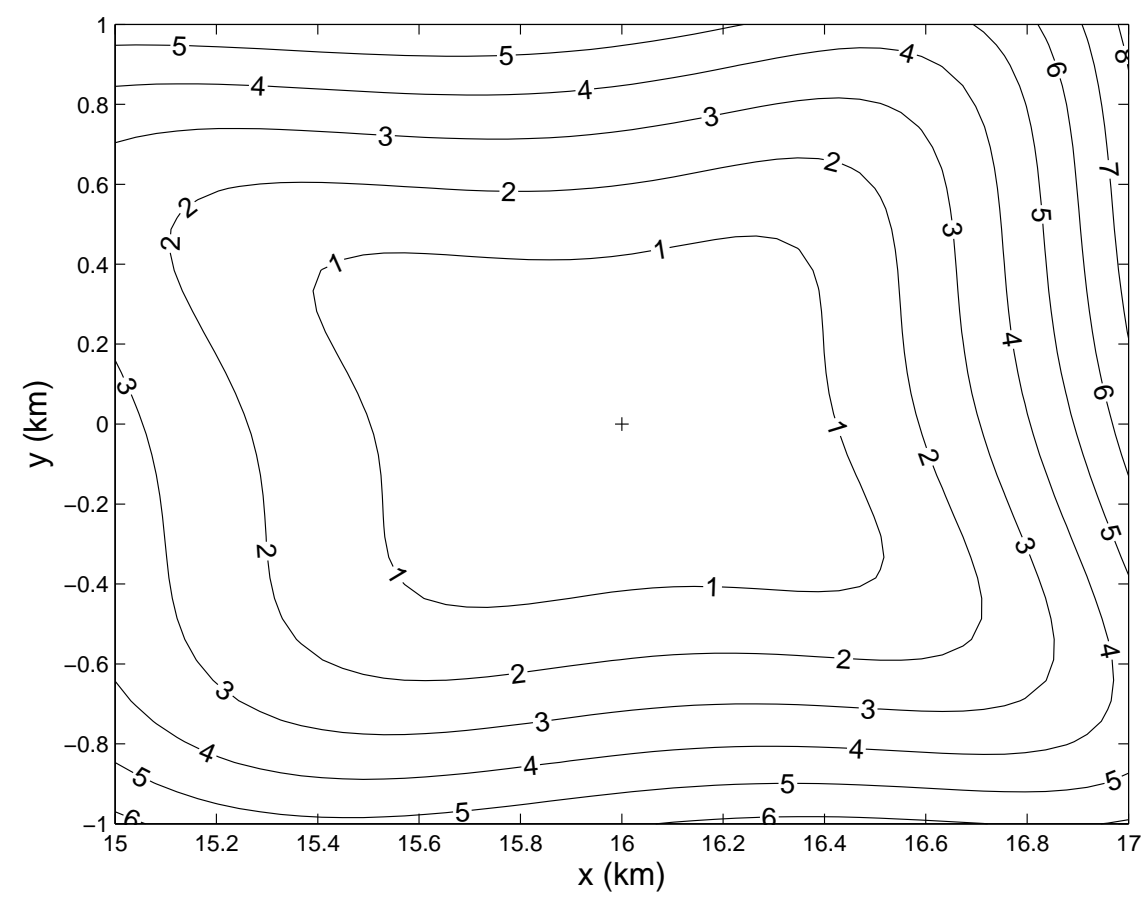

Figure 2. Miss distance $(\mathrm{cm})$ for translation in an XY plane near asteroid Eros 


\section{Sensitivity to Parameter Uncertainty}

Parameter uncertainty is an inevitable part of any space mission. Here, we characterize the effects of parameter uncertainty on our open-loop, constant-thrust translation controller. In our formulation, the final state is dependent on the initial state, rotation rate, thrust, and gravitational potential.

$$
\mathbf{X}_{\mathbf{f}}=g\left(\mathbf{X}_{\mathbf{0}}, \omega, \mathbf{T}, M, C_{n m}, S_{n m}\right)
$$

The parameters $M, C_{n m}$, and $S_{n m}$ represent the body's mass and spherical harmonic coefficients which define the body's potential,

$$
V=\frac{G M}{a} \sum_{n=0}^{\infty} \sum_{m=0}^{n}\left(\frac{a}{r}\right)^{n+1}\left(C_{n m} V_{n m}+S_{n m} W_{n m}\right)
$$

where

$$
V_{n m}=P_{n m}(\sin \phi) \cos m \theta, \quad W_{n m}=P_{n m}(\sin \phi) \sin m \theta,
$$

$a$ is the reference radius, and $\phi$ and $\theta$ are the spacecraft latitude and longitude respectively. $P_{n m}$ are the associated Legendre polynomials. There is uncertainty associated with each of these system parameters. If we expand the perturbed system in a Taylor series, we find:

$$
\delta \mathbf{X}_{\mathbf{f}}=\frac{\partial g}{\partial \mathbf{X}_{\mathbf{0}}} \delta \mathbf{X}_{\mathbf{0}}+\frac{\partial g}{\partial w} \delta w+\frac{\partial g}{\partial \mathbf{T}} \delta \mathbf{T}+\frac{\partial g}{\partial M} \delta M+\sum_{n=1}^{\infty} \sum_{m=0}^{n}\left(\frac{\partial g}{\partial C_{n m}} \delta C_{n m}+\frac{\partial g}{\partial S_{n m}} \delta S_{n m}\right)+\ldots
$$

The partial derivatives, or sensitivity matrices, in Eqn. 14 can be evaluated numerically using standard sensitivity analysis. ${ }^{6}$ If we define the equations of motion:

$$
\dot{\mathbf{X}}=f\left(\mathbf{X}, \omega, \mathbf{T}, M, C_{n m}, S_{n m}\right)
$$

and let $\alpha$ be an uncertain parameter, then the dynamics of the sensitivity matrices are:

$$
\left(\frac{\partial \dot{X}}{\partial \alpha}\right)=\frac{\partial f}{\partial X} \frac{\partial X}{\partial \alpha}+\frac{\partial f}{\partial \alpha}
$$

where

$$
\left.\left(\frac{\partial \mathbf{X}}{\partial \alpha}\right)\right|_{0}=0_{6 x n}
$$

except in the case of sensitivity to initial state, where

$$
\left.\left(\frac{\partial \mathbf{X}}{\partial \mathbf{X}_{0}}\right)\right|_{0}=I_{6 x 6}
$$

Once we know the sensitivity matrices, we can compute the linear covariance of the final state given the covariances of the various parameters,

$$
\operatorname{Cov}\left(\mathbf{X}_{\mathbf{f}}\right)=\Phi\left(t_{f}, 0\right) \operatorname{Cov}\left(\mathbf{X}_{\mathbf{0}}\right) \Phi\left(t_{f}, 0\right)^{T}+\left.\left.\sum_{i=1}^{k} \frac{\partial X}{\partial \alpha_{i}}\right|_{t_{f}} \operatorname{Cov}\left(\alpha_{i}\right) \frac{\partial X}{\partial \alpha_{i}}\right|_{t_{f}} ^{T}
$$

where $\Phi\left(t_{f}, 0\right)$ is the state transistion matrix from time 0 to $t_{f}$.

\section{Sensitivity Results}

Sensitivity of our controller to parameter uncertainty is a function of the time of transfer, endpoints of the trajectory, and body parameters. Instead of trying to cover this entire parameter space, we will look only at low altitude descent trajectories travelling from an outer spherical shell to an inner sphere with the starting position and target having the same latitude and longitude. Uncertainty in any system parameter results in a 6-dimensional hyper-ellipsoid in phase space representing the final state covariance. We will 
concern ourselves only with error in position, parameterized by the square root of the largest eigenvalue of the position covariance matrix,

$$
\sigma=\max \left[\sqrt{\lambda} \in \Re:\left[\lambda I_{3 x 3}-\operatorname{Cov}\left(\mathbf{r}_{f}\right)\right] \hat{v}=0\right]
$$

where $\hat{v}$ is an arbitrary unit vector.

First, we look at sensitivity to errors in the initial state. The sensitivity matrix in this case is equivalent to the state transistion matrix. For descents above a spherical body, we find that errors in initial position are amplified most when descending near the equator and least at the poles. Magnitude of the error in final position is generally on the order of error in the initial position. Assuming the spacecraft uses an in-situ navigation scheme, uncertainty in the initial position should be a few meters. Errors in initial velocity produce the greatest uncertainty in final position for descents near the pole and the least near the equator. Miss due to velocity uncertainty can be large if initial velocity is not well known. Velocity should be known to within a few $\mathrm{mm} / \mathrm{s}$ to keep $\sigma$ on the order of a few meters. When we perform the same descents over an ellipsoidal body, we find that for both initial position and velocity errors, the final position uncertainty is largest at the equator above the elongated ends of the body and smallest at the equator near the body's intermediate semi-major axis. The magnitude of this 'shape effect' dominates the difference between uncertainty in polar and equatorial descents.

We can determine sensitivity to errors in rotation rate using Eqn. 16, where:

$$
\frac{\partial f}{\partial w}=\left[\begin{array}{llll}
0, & 0, \quad 0, \quad 2 \omega x+2 \dot{y}, \quad 2 \omega y-2 \dot{x}, \quad 0
\end{array}\right]^{T}
$$

Generally, uncertainty in $\omega$ is very small. For the Near Earth Asteroid Rendezvous (NEAR) mission, 1-sigma uncertainty in $\omega$ was $3.03 \mathrm{E}-11 \mathrm{rad} / \mathrm{sec}^{7}$ For our class of descents, this uncertainty resulted in $\sigma$ on the order of $10 \mu \mathrm{m}$, which is negligible compared to the effects of other uncertainties. We found that uncertainty in final position was largest for descents near the equator and went to zero for polar descents, which we would expect since $\omega$ has no effect on descent along the $\mathrm{z}$ axis. Again, when the body is elongated, the largest uncertainty in final position occurs above the long ends of the body.

Rotation rate is unique in that it is the only parameter whose sensitivity is directly dependent on initial velocity. We found that radial initial velocities away from the body produce smaller errors than for towards the body and initial velocities in the retrograde direction induce smaller errors than velocities in the direct direction.

Error in the thrust vector, T, results from error in thrust magnitude and error in thrust direction,

$$
\delta T=\delta \theta_{T}\left[\begin{array}{c}
-T_{m a g, 0} \cos \phi_{T, 0} \sin \theta_{T, 0} \\
T_{m a g, 0} \cos \phi_{T, 0} \cos \theta_{T, 0} \\
0
\end{array}\right]+\delta \phi_{T}\left[\begin{array}{c}
-T_{m a g, 0} \sin \phi_{T, 0} \cos \theta_{T, 0} \\
-T_{m a g, 0} \sin \phi_{T, 0} \sin \theta_{T, 0} \\
T_{\text {mag }, 0} \cos \phi_{T, 0}
\end{array}\right]+\delta T_{m a g}\left[\begin{array}{c}
\cos \phi_{T, 0} \cos \theta_{T, 0} \\
\cos \phi_{T, 0} \sin \theta_{T, 0} \\
\sin \phi_{T, 0}
\end{array}\right]
$$

where $\phi_{T}$ and $\theta_{T}$ are the latitude and longitude of the thrust direction respectively, and $T_{\text {mag }}$ is the thrust magnitude. The zero subscript indicates nominal quantities. In Eqn. 16,

$$
\frac{\partial f}{\partial \mathbf{T}}=\left[\begin{array}{l}
0_{3 x 3} \\
I_{3 x 3}
\end{array}\right]
$$

Since thrust is applied in an open-loop manner, miss due to thruster error can be large if the thrust direction and magnitude are not well known. The very low levels of thrust necessary to perform these maneuvers is also a challenge, particularly near very small bodies. The nominal required thrust magnitudes are on the order of $10^{-2}-10^{-5} \mathrm{~N} / \mathrm{kg}$. Wolff et. al. [1998] found in calibration simulations for an ion propulsion system that thrust magnitude can be tuned to an accuracy of $1.2 \%$ with a maximum error of 0.5 $\mathrm{mN}$ and thrust orientation to within 0.5 to 5 degrees. ${ }^{8}$ With this type of calibration, errors due to thruster uncertainty are reasonable.

Error due to thruster uncertainty is the largest contributer to $\sigma$ in almost all cases. Depending on the transfer and the calibration, $\sigma$ due to thrust alone can be from meters to kilometers. The error in final position due to thruster uncertainty as a function of latitude and longitude cannot be easily characterized. For a spherical body, we find that the maximum $\sigma$ due to thrust uncertainty can occur above the equator 
or the pole. Which of these is the case and the relative magnitudes of the errors for a particular situation seem to depend on the transfer time and the time to drop the specified distance without thrust. We find that if the ratio of these times for a particular body is too high or too low, the maximum $\sigma$ will occur at the equator. On the other hand, if this ratio is just about right, we will find maximum $\sigma$ is induced at the pole and minimum error is at the equator. For an ellipsoidal gravity field, the maximum error in the final position occurs for descents either over the intermediate or largest semi-major axis of the body. Generally, for longer transfer times relative to the time to drop freely, we find the largest $\sigma$ for descents above the long ends of the body. For shorter transfer times, we find the largest $\sigma$ for descents above the intermediate semi-major axis. The minimum error occurs at either the poles or the non-maximum equatorial axis depending on the spherical body result.

Finally, we look at sensitivity to errors in the spacecraft's potential model as defined by a spherical harmonic expansion (Eqn. 13). We find that errors in the mass estimate produce the largest errors in final position above the poles and elongated ends of the body. The NEAR mission was able to determine the mass of Eros to within $4.5 E-2 \% .^{7}$ Using the same percentage error, we found errors due to mass uncertainty to be on the order of centimeters or better.

Errors in the $C_{10}, C_{11}$, and $S_{11}$ coefficients correspond to offsets of the center of mass in the x, y, and z directions respectively. If we look at uncertainty in each term individually, each causes maximum uncertainty in final position for descents along its respective axis. When all three are considered concurrently, we find that error is maximum for polar descents and minimum for equatorial descents. Using the 1-sigma uncertainty in these coefficients found by the NEAR mission $(4 E-6)$, errors in final position for our descents were on the order of millimeters.

Looking at the higher order harmonics, a general pattern emerges. For the $C_{n m}$ coefficient, $\sigma$ for these descents is proportional to $V_{n m}(\phi, \theta)$. Similarly, the error due to uncertainty in the $S_{n m}$ coefficient is proportional to $W_{n m}(\phi, \theta)$. This is not surprising since the sensitivity equation is driven by $\frac{\partial^{2} V}{\partial \mathbf{r} \partial\left[C_{n m}, S_{n m}\right]}$ and the spacecraft moves with nearly constant latitude and longitude. The one-sigma errors that arise from uncertainties in the harmonic coefficients are generally on the order of meters or less.

When all these parameters are considered simultaneously, we found uncertainty in the final position is likely to be largest for descents above the equator of an elongated body, either near the longest or intermediate semi-major axis. Descents with minimum uncertainty in final position may occur above the equatorial semimajor axis not corresponding to the maximum error or above the poles depending on the distance and duration of the transfer. For a nearly spherical body, it is possible that the largest values of $\sigma$ will be found for descents above the poles. Thruster error is the dominant contributer to $\sigma$, followed by error in the initial state.

\section{Covariance Simulations}

We have performed numerical covariance analyses, including uncertainty in all of the parameters above, of descent trajectories at a range of latitudes and longitudes above three realistic asteroid shapes. We consider triangular faceted polyhedral models of the asteroids Itokawa and Eros, as well as an ellipsoidal approximation of the asteroid Vesta. For each body, we will implement three different sets of parameter covariances given in Table 1.

For all three covariance sets, we assume the spacecraft uses in-situ measurements (i.e. optical navigation and/or altimetry) in addition to Doppler range and range rate measurements to refine its position estimate to the given level. We assume the thruster (electric propulsion or low-thrust chemical) has been calibrated in the manner discussed by Wolff et. al., ${ }^{8}$ though we do not impose a maximum error in the thrust magnitude. The one-sigma values given for covariance set \#1 correspond to a mission with a well-calibrated thruster and advanced navigation instruments, while the accuracy in set \#3 may be obtained by a cheaper mission. The sigmas we use for $\omega$, mass, and the harmonic coefficients are those found during the Near Earth Asteroid Rendezvous (NEAR) Mission. ${ }^{7}$ We will assume other visiting spacecraft can characterize their target to this level of certainty.

\section{A. Itokawa}

The first small-body we look at is asteroid Itokawa, the target of the currently en route Japanese Hayabusa spacecraft. Itokawa is relatively small, measuring only 548 x 312 x $276 \mathrm{~m}$, and is very nearly a perfect 


\begin{tabular}{||l|c|c|c||}
\hline Covariance Set & $\# 1$ & $\# 2$ & $\# 3$ \\
\hline \hline Position $(\mathrm{x}, \mathrm{y}, \mathrm{z})$ & $1 \mathrm{~m}$ & $5 \mathrm{~m}$ & $10 \mathrm{~m}$ \\
\hline Velocity $(\mathrm{x}, \mathrm{y}, \mathrm{z})$ & $0.1 \mathrm{~mm} / \mathrm{s}$ & $1 \mathrm{~mm} / \mathrm{s}$ & $5 \mathrm{~mm} / \mathrm{s}$ \\
\hline$\theta$ & $0.5^{\circ}$ & $2^{\circ}$ & $5^{\circ}$ \\
\hline$\phi$ & $0.5^{\circ}$ & $2^{\circ}$ & $5^{\circ}$ \\
\hline$T_{\text {mag }}$ & $0.1 \%$ & $0.8 \%$ & $2.0 \%$ \\
\hline$\omega$ & $3.03 E-11 \mathrm{rad} / \mathrm{s}$ & $3.03 E-11 \mathrm{rad} / \mathrm{s}$ & $3.03 \mathrm{E}-11 \mathrm{rad} / \mathrm{s}$ \\
\hline Mass & $0.0448 \%$ & $0.0448 \%$ & $0.0448 \%$ \\
\hline 1st Order Harmonics & $4 E-6$ & $4 E-6$ & $4 E-6$ \\
\hline 2nd Order Harmonics & $4 E-6$ & $4 E-6$ & $4 E-6$ \\
\hline 3rd Order Harmonics & $5 E-6$ & $5 E-6$ & $5 E-6$ \\
\hline
\end{tabular}

Table 1. One-sigma parameter uncertainties for descent simulations

ellipsoid. The rotation period of Itokawa is 12.132 hours and its bulk density is approximately $2.5 \mathrm{~g} / \mathrm{cm}^{3}$. For these runs, the spacecraft will descend from an outer sphere with radius $600 \mathrm{~m}$ to an inner sphere of radius $310 \mathrm{~m}$ in 1200 seconds. A 6098 vertex polyhedron was used to model the body's potential. ${ }^{5}$

The results of these simulations for the three covariance sets in Table 1 are shown in Table 2 . The largest and smallest $\sigma$ values and their locations are given. For all three bodies, descents were done every $9^{\circ}$ latitude and longitude, so the locations given are accurate only to $9^{\circ}$ resolution. The area averaged $\sigma$ is also given. Figure 3 shows $\sigma$ as a function of longitude and latitude for descents using covariance set \#3. The effect of longitude and latitude on descents using the other covariance sets are similar.

\begin{tabular}{||c|c|c|c||}
\hline Covariance Set & $\operatorname{Max} \sigma(m)\left(\operatorname{Lat} / \operatorname{Long}\left({ }^{\circ}\right)\right)$ & $\operatorname{Min} \sigma(m)\left(\operatorname{Lat} / \operatorname{Long}\left(^{\circ}\right)\right)$ & Avg $\sigma(m)$ \\
\hline$\# 1$ & $2.704(0,-90)$ & $2.630(90,-)$ & 2.650 \\
\hline$\# 2$ & $11.302(0,-90)$ & $11.015(90,-)$ & 11.091 \\
\hline$\# 3$ & $27.733(0,-90)$ & $27.004(90,-)$ & 27.207 \\
\hline
\end{tabular}

Table 2. Results of covariance analysis of descents above Itokawa

Here, we see that the largest uncertainty in final position occurs above the body's intermediate semimajor axis at the equator. This maximum is attributable to error due to thrust uncertainty. For Itokawa, the duration of our transfer relative to the distance covered is short enough that the maximum error occurs here. The secondary effect of the remaining parameter uncertainties causes $\sigma$ to increase above the body's largest semi-major axis. Polar descents yield the minimum uncertainty in final position for this transfer. We do not see too much variation in $\sigma$ over Itokawa for these descents, with less than $3 \%$ difference between the maximum and minimum for all covariance sets.

Considering no feedback is used, the magnitude of errors induced by covariance sets \#1 and \#2 are quite reasonable. One reason for this is that the necessary thrust magnitude for these maneuvers is so small; errors in thrust magnitude and orientation do not push the spacecraft off course very strongly. However, it may be difficult for an actual spacecraft to reliably implement these very low thrust levels. The $27 \mathrm{~m}$ average $\sigma$ value from set $\# 3$ is also small, but corresponds to about $5^{\circ}$ angular error, which may be significant.

The authors would like to correct the results for covariance of descents above Itokawa given in Broschart and Scheeres. ${ }^{9}$ The figure and discussion for the Itokawa descent results were incorrect. The data presented was for ascending trajectories. The discussion here is correct and should replace the previous result.

\section{B. Eros}

The asteroid Eros was recently visited by the NEAR spacecraft. The first and only successful descent to an asteroid was performed by this spacecraft using a series of open-loop burns to descend from orbit. ${ }^{1}$ In these descent simulations, we use an 809 vertex shape model of Eros measuring approximately $32.8 \times 14.4$ 


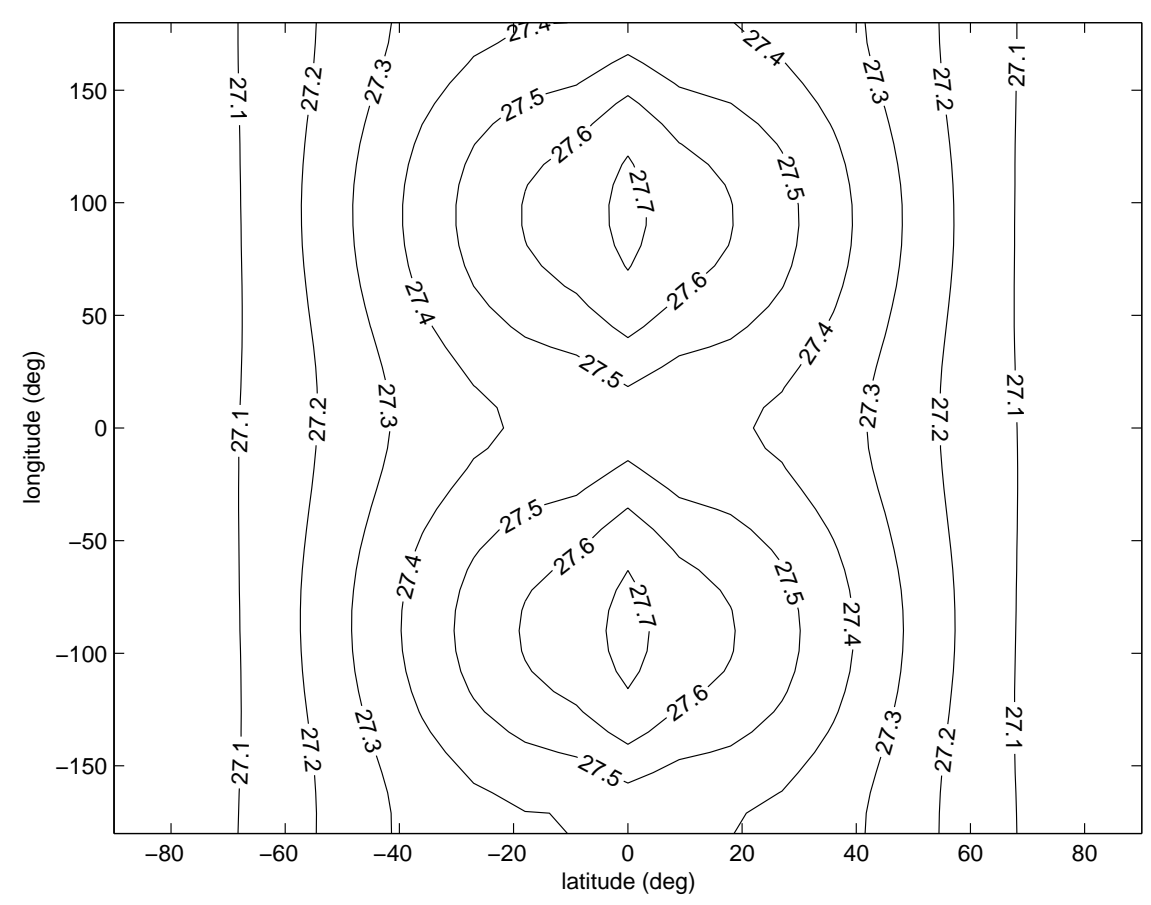

Figure 3. $\sigma(m)$ as a function of latitude and longitude for radial descent over asteroid Itokawa

$\mathrm{x} 11.9 \mathrm{~km}$. The orbit period of Eros is 5.27 hours and its bulk density is approximately $2.67 \mathrm{~g} / \mathrm{cm}^{3}$. The shape of Eros, shown in figure 4, is quite elongated with two large craters on either side of its midsection. We present results here for descents from an outer sphere of radius $16.2 \mathrm{~km}$ to an inner sphere of $15.7 \mathrm{~km}$ in 1500 seconds.

The results of these simulations are shown in Table 3 . We can see there is a large difference between maximum and minimum $\sigma$, with the maximum being nearly 5 times the minimum. This is attributable to Eros' elongated shape and varied surface. The minimum value occurs above one of the large craters on the body's side and the maximum occurs at the most elongated end of the body. The polar area is also a relatively good place to land. Figure 5 shows $\sigma$ as a function of latitude and longitude for these descents using covariance set \#2. Descents above most of the surface produce a $\sigma$ of between 30 and 40 meters. Near the long ends of the body, uncertainty quickly grows large and there is some improvement near the large craters. The average errors for descents to Eros seem reasonable considering the size of the body. The maximum $\sigma$ obtained with covariance set \#3 corresponds to a miss of only $0.9^{\circ}$ and the average is only $0.36^{\circ}$. With improved calibration and parameter certainty, results are even better.

\begin{tabular}{||c|c|c|c||}
\hline Covariance Set & $\operatorname{Max} \sigma(m)\left(\operatorname{Lat} / \operatorname{Long}\left({ }^{\circ}\right)\right)$ & $\operatorname{Min} \sigma(m)\left(\operatorname{Lat} / \operatorname{Long}\left(^{\circ}\right)\right)$ & $\operatorname{Avg} \sigma(m)$ \\
\hline$\# 1$ & $24.816(-9,-171)$ & $5.280(0,36)$ & 10.315 \\
\hline$\# 2$ & $99.597(-9,-171)$ & $21.585(0,36)$ & 41.536 \\
\hline$\# 3$ & $249.024(-9,-171)$ & $53.319(0,36)$ & 103.716 \\
\hline
\end{tabular}

Table 3. Results of covariance analysis of descents above Eros

\section{Vesta Ellipsoid}

Finally, we look at descents above a very large asteroid, Vesta. We approximate the true shape of Vesta by a tri-axial ellipsoid with semi-major axes of 289, 280, and $229 \mathrm{~km}$. Thomas et. al. approximated the density of Vesta to be $3.7 \mathrm{~g} / \mathrm{cm}^{3}$ and its rotation rate to be 5.342 hours. ${ }^{10}$ Here, we present results for a 1800 second radial descent covering $10 \mathrm{~km}$ from a radius of $300 \mathrm{~km}$ to $290 \mathrm{~km}$. The results of these simulations are given in Table 4 and $\sigma$ as a function of longitude and latitude for covariance set \#2 is shown in figure 6 . 


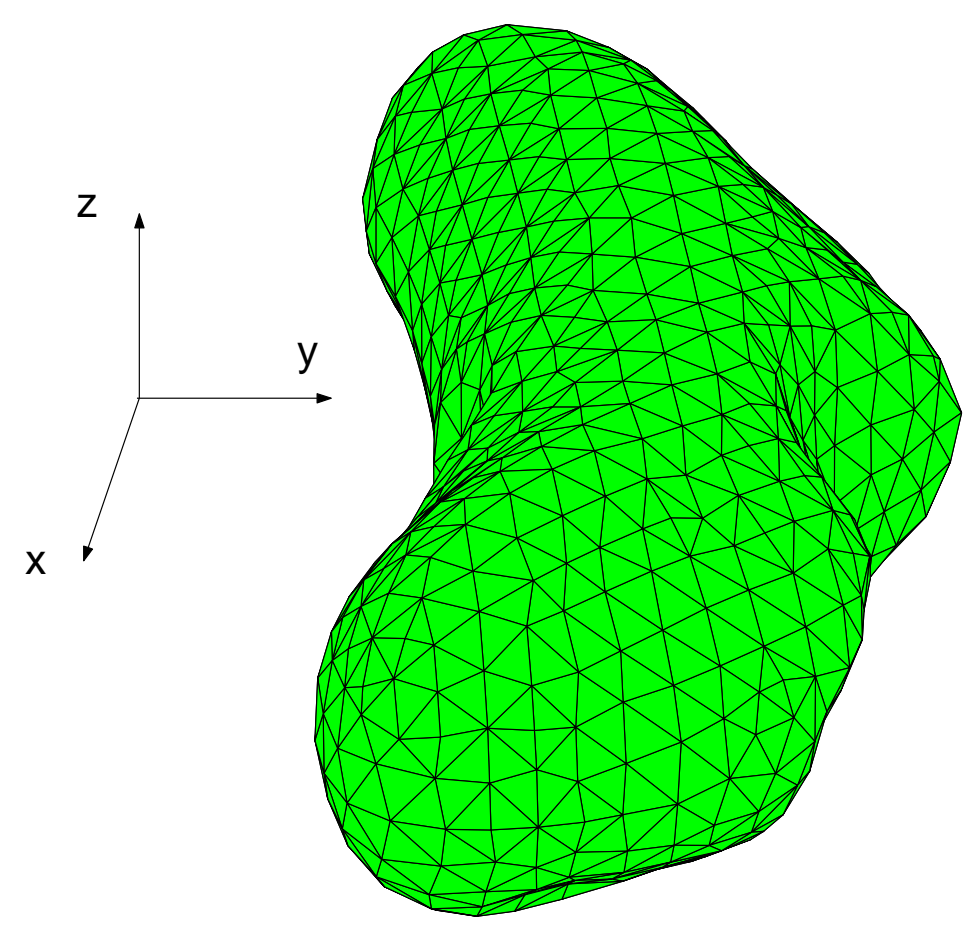

Figure 4. 809 Vertex Polyhedral Model of the Asteroid Eros

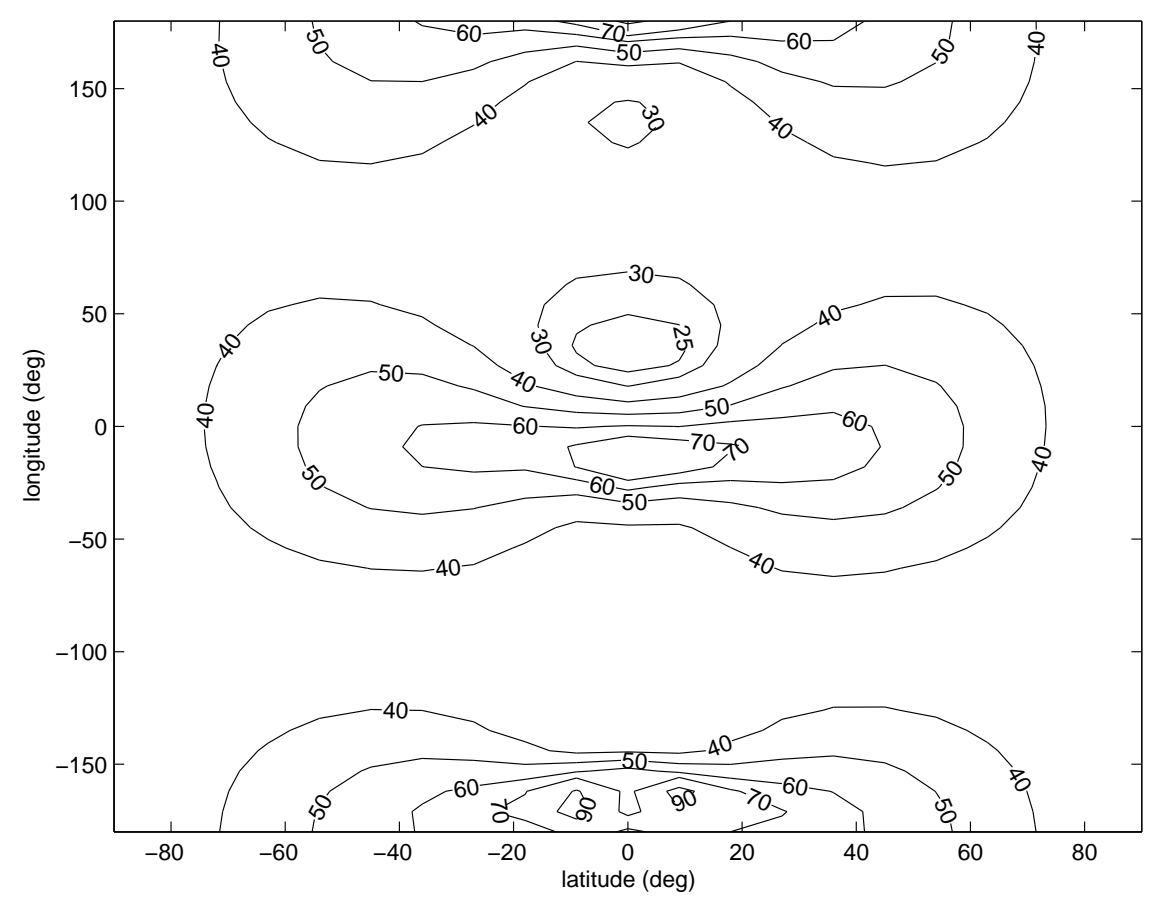

Figure 5. $\sigma(m)$ as a function of latitude and longitude for radial descent over asteroid Eros 


\begin{tabular}{||c|c|c|c||}
\hline Covariance Set & $\operatorname{Max} \sigma(m)\left(\operatorname{Lat} / \operatorname{Long}\left({ }^{\circ}\right)\right)$ & Min $\sigma(m)\left(\operatorname{Lat} / \operatorname{Long}\left(^{\circ}\right)\right)$ & Avg $\sigma(m)$ \\
\hline$\# 1$ & $2469.9(0,180)$ & $2104.5(-18,-90)$ & 2113.2 \\
\hline$\# 2$ & $9989.6(0,180)$ & $8444.9(-18,-90)$ & 8559.8 \\
\hline$\# 3$ & $24977(0,180)$ & $21116(-18,-90)$ & 21403 \\
\hline
\end{tabular}

Table 4. Results of covariance analysis of descents above a $289 \times 280 \times 220 \mathrm{~km}$ ellipsoid (approximation of asteroid Vesta)

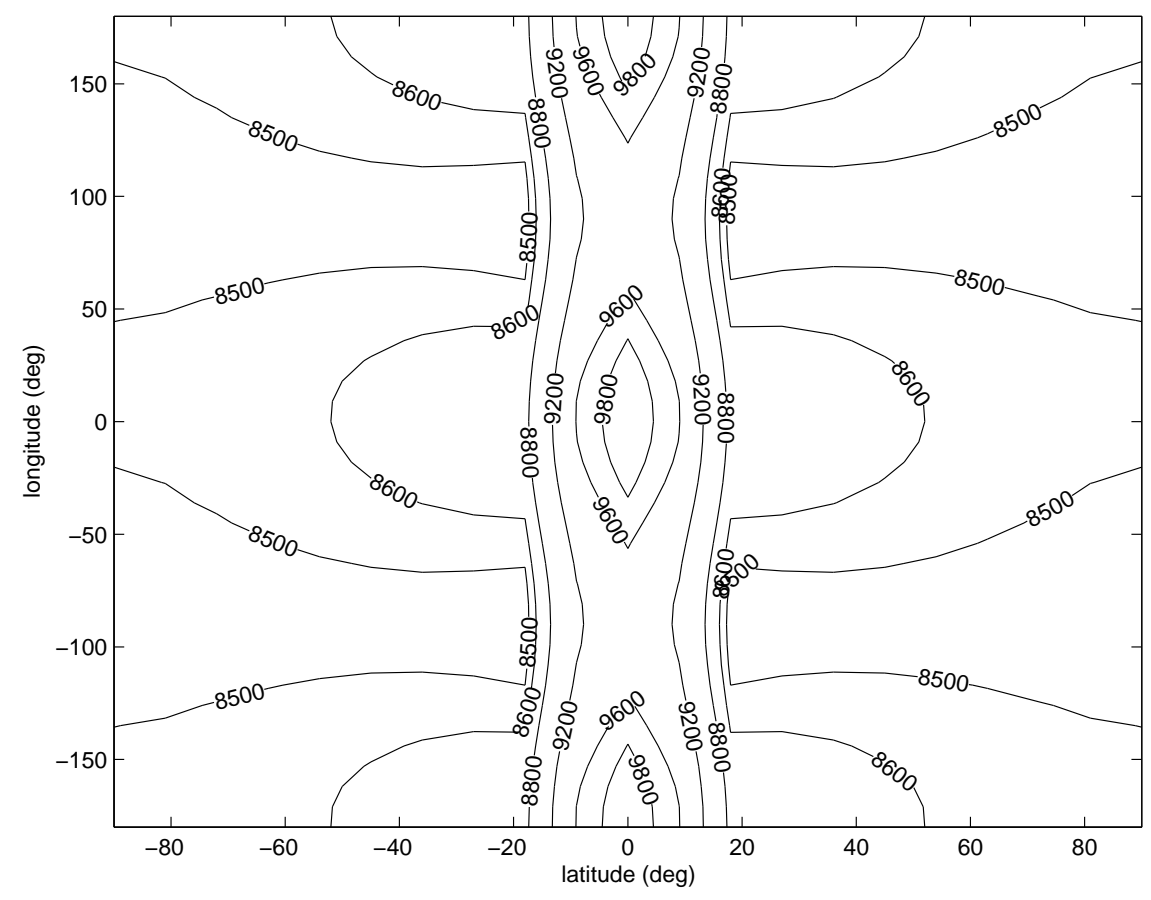

Figure 6. $\sigma(m)$ as a function of latitude and longitude for radial descent over a $289 \times 280 \times 220 \mathrm{~km}$ ellipsoid (approximation of asteroid Vesta) 
Errors due to parameter uncertainty in these descents are quite large. These errors are primarily due to thruster orientation errors. Because the necessary thrust is so large, any misalignment causes a significant perturbing force, which goes unchecked because of the nearly constant gravitational attraction in the immediate vicinity of the spacecraft.

In reality, a constant thrust translation approach would probably be a poor choice for a body as large as Vesta. A large amount of fuel would be necessary to perform constant thrust maneuvers and impact velocity for a free-drop or constant thrust descent would be high.

\section{Detailed Descent Scenario}

Now we will take a more detailed look at a descent using the constant thrust and free-drop solutions. The descent described here is very similar to the final phase of the NEAR mission descent. ${ }^{1}$ The spacecraft will descend from roughly $2.25 \mathrm{~km}$ altitude to touchdown in the vicinity of the Himeros depression on the asteroid Eros (modeled by our 809-vertex polyhedron). The spacecraft will begin at ( $2.25 \mathrm{~km}$ altitude, $-35^{\circ}$ latitude, $82^{\circ}$ longitude) with about $70 \%$ of orbital speed $(3 \mathrm{~m} / \mathrm{s}$ retrograde in the body-fixed frame). A constant thrust segment will then slow the spacecraft to arrival at $\left(156 \mathrm{~m},-30^{\circ}, 60^{\circ}\right)$. The spacecraft will then drop without thrust to the surface target at $\left(0 \mathrm{~m},-29.345^{\circ}, 61.123^{\circ}\right)$. The first maneuver will be performed in 40 minutes and the free-drop segment will take 206 seconds.

Given the initial state and the target position, we use eqns. 7 and 11 to obtain the corrected constant thrust value, $[-0.557 \mathrm{mN} / \mathrm{kg}(\mathrm{x}), 4.259 \mathrm{mN} / \mathrm{kg}(\mathrm{y}),-2.542 \mathrm{mN} / \mathrm{kg}(\mathrm{z})]$, to complete the segment. Eqn. 8 , corrected similarly to the thrust, gives the predicted velocity at the waypoint. We then use Eqn. 5 to numerically determine the time to free-drop from the predicted state to the surface. The free-drop trajectory cannot be corrected through error estimate because the target state cannot be freely changed without thrust.

Figure 7 shows the planned path (waypoints indicated by circles) and the actual trajectory of the descent. The dashed line is the powered portion and the solid line is the free-drop trajectory. The nominally miss distance at the first waypoint for our controller was $11.39 \mathrm{~m}$. The nominal miss distance at the touchdown point was $20.65 \mathrm{~m}$. The touchdown velocity was $1.165 \mathrm{~m} / \mathrm{s}$ normal to the surface and $1.230 \mathrm{~m} / \mathrm{s}$ tangent to the surface. When the uncertainties in covariance set \#2 were included, we found the 1-sigma ellipsoid to have semi-major axes of $374.90 \mathrm{~m}, 346.71 \mathrm{~m}$, and $185.65 \mathrm{~m}$ at the thrust cut-off waypoint and $375.20 \mathrm{~m}$, $346.79 \mathrm{~m}$, and $190.67 \mathrm{~m}$ at the touchdown point. On the ground, these errors correspond to a few degrees longitude or latitude error, which may be acceptable. However, the 1-sigma error at the waypoint is larger than the nominal altitude. Without measurements, the waypoint must be at a higher altitude, which means a larger impact velocity. Errors in thrust application account for most of the uncertainty we find. Without it, the 1-sigma ellipsoid at the target is $69.04 \times 28.88 \times 9.94 \mathrm{~m}$.

\section{Conclusions}

We have developed a closed-form solution to the small-body equations of motion using linearization and a constant thrust assumption. This solution solves for the free-drop surface, which is the locus of initial states that result in interception of a target position without thrust. The necessary constant thrust to complete a point to point translation is also formulated. The constant thrust translation solution is improved by including an error estimate calculated using the linearized trajectory and the spacecraft's internal potential model. We have found this controller to have nominal accuracy to the order of centimeters for transfers in the vicinity of the initial position.

We have also formulated the sensitivity equations for various system parameters. We found that errors in thrust and initial state have the greatest impact on error in the final position. Numerical examples of descent uncertainty above realistic asteroid shapes have been presented. A detailed discussion of a descent scenario to asteroid Eros was also given.

\section{Acknowledgements}

The research described in this paper was sponsored by the Interplanetary Network Technology Program by a grant to The University of Michigan from the Jet Propulsion Laboratory, California Institute of Technology which is under contract with the National Aeronautics and Space Administration. S. Broschart was also supported by the Graduate Student Researchers Program at the Jet Propulsion Laboratory and by the Michigan Space Grant Consortium. 


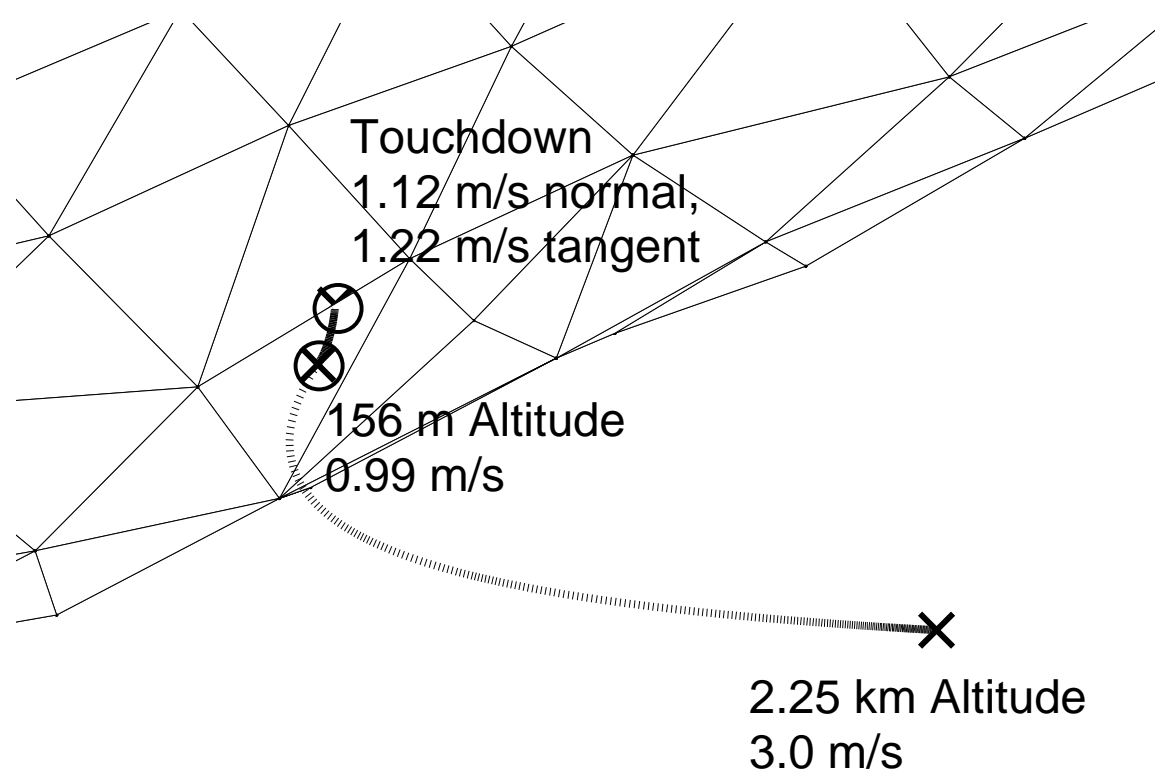

Figure 7. Descent to the surface of asteroid Eros using constant thrust and free-drop control

\section{References}

${ }^{1}$ Dunham, D.W., Farquhar, R.W., McAdams, J.V., Holdridge, M., Nelson, R., Whittenburg, K., Antreasian, P., Chesley, S., Helfrich, C., Owen, W.M., Williams, B., Veverka, J., and Harch, A., "Implementation of the first asteroid landing", Icarus, Vol. 159, No. 2, 2002, pp. 433-438.

${ }^{2}$ Cui, H.T. and Cui, P.Y., "Autonomous navigation and guidance for soft-landing asteroid", Journal of Astronautics (1000-1328), Vol. 23, No. 5, 2002, pp. 1-4.

${ }^{3}$ Guelman, M. and Harel, D., "Power limited soft landing on an asteroid", AIAA Journal of Guidance, Control and Dynamics, Vol. 17, No. 1, 1994, pp. 15-20.

${ }^{4}$ Kubota, T., Hashimoto, T., Sawai, S., Kawaguchi, J., Ninomiya, K., Uo, M., and Baba, K., "An autonomous navigation and guidance system for MUSES-C asteroid landing", Acta Astronautica (0094-5765), Vol. 52, No. 2-6, 2003, p. 125-131

${ }_{5}^{5}$ Ostro, S.J., Benner, L.A.M., Nolan, M.C., Magri, C., Giorgini, J.D., Scheeres, D.J., Broschart, S.B., Kaasalainen, M., Vokrouhlicky, D., Chesley, S.R., Margot, J., Jurgens, R.F., Rose, R., Yeomans, D.K., Suzuki, S., De Jong, E.M., "Radar Observations of Asteroid 25143 Itokawa (1998 SF36)", Meteoritics and Planetary Science, Vol. 39, No. 3, pp. 407-424.

${ }^{6}$ Montenbruck, O., Gill, E., "Satellite Orbits, Models, Methods, and Applications", Springer-Verlag Berlin Heidelberg, 2nd printing, 2001, pp. 241-243.

${ }^{7}$ Miller, J.K., Konopliv, A.S., Antreasian, P.G., Bordi, J.J., Chesley, S., Helfrich, C.E., Owen, W.M., Wang, T.C., Williams, B.G., Yeomans, D.K., "Determination of Shape, Gravity, and Rotational State of Asteroid 433 Eros", Icarus, Vol. 155, No. 3-17.

${ }^{8}$ Wolff, P.J., Pinto, F., Williams, B.G., Vaughan, R.M., "Navigation Considerations for Low-thrust Planetary Missions", AAS 98-201, AAS/AIAA Spaceflight Mechanics Meeting, Monterey, CA, Feburary 9-11, 1998.

${ }^{9}$ Broschart, S.B., Scheeres, D.J., "Spacecraft Descent and Translation Near Small-Bodies", 24th International Symposium on Space Technology and Science, Miyazaki, Japan, May 31-June 6, 2004, ISTS 2004-d-35.

${ }^{0}$ Thomas, P.C., Binzel, R.P., Gaffey, M.J., Storrs, A.D., Wells, E., Zellner, B.H., "Impact Excavation on Asteroid 4 Vesta: Hubble Space Telescope Results", Science 277, pp.1492-1495, 1997. 